\title{
Estilos de vida de los clientes y calidad de servicio de la asociación de comerciantes agro artesanal San Borja
}

\author{
Dora Alejandrina Polo Cerna
}

Universidad Nacional Federico Villarreal

\section{RESUMEN}

La presente investigación titulada: Estilos de vida de los clientes y calidad de servicio de la asociación de comerciantes agro artesanal de San Borja (ACMSB), tuvo como objetivo general determinar la relación que existe entre los estilos de vida de los clientes y la calidad de servicio de los empresarios de la ACMSB; los objetivos específicos fueron: Identificar los estilos de vida de los clientes; conocer la percepción de los clientes respecto a la calidad de servicio de los empresarios de la ACMSB. El método general utilizado fue el método científico, los instrumentos fueron dos cuestionarios Servqual y estilos de vida. La muestra compuesta de 171 clientes, varones y mujeres mayores de 18 años, cuyo consumo supero los S/.10.00 y que el día de la encuesta se encontraban comprando bienes y servicios en las instalaciones de la ACMSB, se incluyeron a las empleadas del hogar y se excluyeron a clientes mayores de 80 años. La hipótesis fue: Existe relación entre estilos de vida y la calidad de servicio de los clientes de la ACMSB. Para el análisis de datos se hizo uso de la estadística descriptiva y para la prueba de hipótesis el análisis inferencial. La prueba de Pearson arroja 0.746 que significa que existe una relación de $74 \%$ entre calidad de servicio y estilos de vida de los clientes de la asociación de comerciantes agro artesanal del San Borja. El estilo de vida de los clientes de la ACMSB es el moderno; este resultado coincide con los hallazgos de Arellano [1] quien reporto que el estilo de vida moderno es el preponderante en nuestro país. El hallazgo encontrado por De Soto [2] que los recién llegados del campo a la ciudad, encontraron un muro impenetrable de reglas, que los marginaban de las actividades sociales y económicas legalmente establecidas, en los que era sumamente difícil entrar a negocios formales, se ha logrado superar; puesto que estas micro y pequeñas empresas son viables, legales y están siendo muy bien vistos por los clientes. Mayor argumento constituye el reporte estadístico del INEI [3], que informa que la actividad comercial tuvo un crecimiento del 10,96 \%, esto es una tendencia positiva en la mayoría de sus rubros componentes, destacándose entre otros, la venta minorista de alimentos, a través de las cadenas de supermercados e hipermercados y grandes almacenes. Se concluye que las empresas del sector retail en especial de la ACMSB, están reinventando estrategias en torno a la satisfacción del cliente y son conscientes de la invasión de las grandes cadenas de supermercados e hipermercados y grandes almacenes. Esta investigación servirá como base para futuras estrategias a adoptar por los empresarios de la ACMSB, a fin de mantener a los clientes satisfechos, con alta calidad de servicio.

Descriptores: estilos de vida, clientes, calidad de servicio, asociación de comerciantes agro artesanal San Borja.

\section{ABSTRACT}

This research entitled: Lifestyle and quality customer service the business association artisanal agro San Borja (ACMSB), generally aimed at determining the relationship between the lifestyles of our customers and quality service employers in the ACMSB, the specific objectives were: To identify the lifestyles of our customers, know the customer perception about the quality of service for entrepreneurs ACMSB. The general method used was the scientific method, the instruments were two questionnaires Servqual and lifestyles. The sample consists of 171 clients, men and women over 18, whose consumption exceeded the S/.10.00 and the day of the survey were buying goods and services ACMSB facilities are included domestic workers and excluded clients over 80 years. The hypothesis was: There is a relationship between lifestyle and quality of service for customers ACMSB. For data analysis was done using descriptive statistics and hypothesis testing for inferential analysis. Pearson test sheds 0,746 which mean there is a ratio of $74 \%$ between service quality and lifestyles of customers in the traditional agro business association of San Borja. The lifestyle of the customers is the modern ACMSB, this result agrees with the findings of Arellano [1] who reported that the modern lifestyle is 
preponderant in our country. The finding found by De Soto [2] that the newcomers from the countryside to the city, found an impenetrable wall of rules, that marginalized social and economic activities legally established, in which it was extremely difficult to get into formal business was has been overcome, since these micro and small businesses are viable, legal, and are still very well regarded by customers. Major argument is the statistical report INEI [3], which reports that business grew by $10.96 \%$, this is a positive trend in most of its component items, highlighting among others, retail food through supermarkets and hypermarkets and department stores. It concludes that companies in the retail sector especially the ACMSB are reinventing strategies around customer satisfaction and are aware of the encroachment of large chains of supermarkets and hypermarkets and department stores. This research will serve as a basis for future strategies to be adopted by employers in the ACMSB, in order to keep customers satisfied with high quality service.

Keywords: lifestyle, customers, service quality, artisanal agro business association San Borja.

\section{INTRODUCCIÓN}

En nuestro país, como en México, Colombia, Chile, Argentina, entre otros; en los últimos diez años se ha observado alta competitividad en el sector retail, la penetración de cadenas de supermercados e hipermercados, centros comerciales, power centers, entre otros; están compitiendo fuertemente, al extremo de hacer quebrar a algunos retaileres (tipo de negocios de consumidores finales, conocido como comercio) tradicionales como los que existen también en nuestro medio, que subsisten como asociaciones de comerciantes, mercados cooperativos, mercados de productores, entre otros.

La traducción de retail en el diccionario es: "Ventas al detalle", "revender". El comercio detallista (retailers) está constituido por todas las actividades que intervienen en la venta de bienes y/o servicios, directamente a los consumidores, para su uso personal no lucrativo.

Los negocios tipo retail abarcan desde la tienda de nuestro barrio, hasta las grandes multitiendas e hipermercados, no es un término privativo de este último segmento, sino que es posible aplicar esta conceptualización a pequeñas tiendas o bodegas, almacenes de barrio, el kiosco de la esquina y el mini market del barrio. Por tanto, la gran mayoría de los negocios retail son micro, pequeñas y medianas empresas (Mypes y Pymes).

En el Perú, el segmento retail establecido en Lima y en las ciudades más importantes, comprende tottus, sodimac, mall aventura plaza, supermercados peruanos (del grupo Interbank: plaza vea) wong, grupo falabela, entre otros.
De Soto (2009) en su obra el misterio del capital, señala que "en Perú, los recién llegados del campo a la ciudad, encontraron un muro impenetrable de reglas que los marginaban de las actividades sociales y económicas legalmente establecidas, era sumamente difícil, para estos nuevos citadinos adquirir viviendas legales, entrar a negocios formales o encontrar un empleo legal" (p.27).

Este es el caso de los empresarios de la asociación de comerciantes del mercado de San Borja, quienes realizan sus operaciones de comercio en el distrito de San Borja, pero radican en los distritos de Villa el Salvador, Villa María del Triunfo, San Juan de Miraflores, San Juan de Lurigancho, entre otros.

INEI (2010) declara:

La actividad comercial tuvo un crecimiento de $10.96 \%$, por el dinamismo que mostro la venta y reparación de vehículos y el crecimiento del comercio al por mayor y al por menor; en el que destacaron la venta al por mayor de materiales de construcción y artículos de ferretería, maquinaria, equipo y productos intermedios, la venta minorista de alimentos, bebidas, y tabaco, productos farmacéuticos, enseres domésticos, productos textiles, prendas de vestir y calzado y la venta a través de las cadenas de supermercados $e$ hipermercados y grandes almacenes. En lo que va del año, la actividad comercial viene mostrando tendencia positiva en la mayoría de sus rubros componentes (ver 
anexo $\mathrm{N}^{\circ}$ 3: Ventas reales del sector comercio 2010).

El distrito de San Borja, fue creado el 01 de junio de 1983, localizado dentro de la zona de Lima Centro o Lima tradicional, delimita por el norte con los distritos de la Victoria y San Luís; por el este con Ate y Surco; por el sur con Surco y Surquillo; por el oeste con Surquillo y San Isidro.

Su población asciende a 105, 430 habitantes (según INEI- Censo del año 2007); quienes cuentan para la provisión de bienes de consumo dentro de su jurisdicción con tiendas Wong, supermercados peruanos: plaza vea, y metro; quienes compiten con empresarios del comercio organizados en asociaciones de comerciantes, mini markets, bodegas y mercados como el mercado de San Borja (motivo de la presente investigación).

Según el plan estratégico 2007 - 2010, de la Municipalidad de San Borja, el objetivo general es promover la formalización y desarrollo de Mypes y Pymes sostenibles, construyendo un marco legal que incremente la formalización de comercios informales en un $20 \%$ (5\% por año) y brinden sus servicios al distrito; poner en marcha el programa de capacitación para vecinos emprendedores.

Arellano, (2005) realiza un análisis integral de Lima actual, indaga en sus deseos, sus convicciones, sus comportamientos, sus esperanzas y sus proyectos. Muestra como en solo 30 años sin que los limeños clásicos se dieran cuenta, Lima creció llegando a contener $2 / 3$ de la población de la ciudad. Además muestra que esas zonas son mucho menos pobres de lo que se pensaba, error que resultaba de considerar solamente su ingreso monetario (mostrado en las cifras oficiales) y no ver el menor costo de vida en la zona, ni la riqueza acumulada en bienes patrimoniales que, además de dar mejor calidad de vida, evita el gasto de pago de alquileres.

Arellano (2008) "muestra la existencia de 9 estilos de vida: las conservadoras, los tradicionales, los progresistas, los sobrevivientes, las trabajadoras, los adaptados, los afortunados, los emprendedores y los sensoriales". La identificación de estos estilos de vida permitirá conocer a los consumidores de la ACMSB y en base a ello promover de una manera efectiva, mejores productos y servicios, más clientes satisfechos, más valor para las empresas, y mejoras en los sistemas de comercialización para sus usuarios.

Según Arellano (2010), desde la primera vez en que Arellano Marketing identificara y describiera los estilos de vida en nuestro país, han transcurrido once años y, en comparación con las cifras actuales, la diferencia, aunque no es abismal, sí es importante y explica mucho de lo que está sucediendo hoy. En 1996 los estilos de vida modernos representaban al $42.6 \%$ de la población nacional, para el 2005 concentraban al $52 \%$ de los peruanos. Por eso es que la llegada de estos nuevos formatos a las provincias peruanas, no corresponde tanto a una estrategia de imponer una oferta moderna a una población no interesada, sino más bien viene a responder a una demanda insatisfecha de modernidad que crece.

Con el esquema tradicional de segmentación por niveles socioeconómicos un panorama como el actual, en el que nuevos segmentos de la población demandan productos y servicios cada vez más sofisticados, no hubiese sido detectado con mucha precisión, dado que un mayor ingreso no implica un deseo de mayor modernidad. La visión del mercado desde la perspectiva de los estilos de vida ha hecho evidentes éstas y muchas otras oportunidades como por ejemplo, el consumo en la nueva Lima o la influencia y las expectativas de los peruanos en el exterior. Esto se explica porque la segmentación, en el caso de los estilos de vida, no es horizontal, sino vertical. En este orden de ideas, el volumen de quienes estén dispuestos (por aspiración) a comprar será mayor al de aquellos que puedan (por capacidad adquisitiva) hacerlo.

En relación a los estilos de vida entre la medición de 1996 y la que se maneja actualmente se ha pasado de nueve a seis estilos de vida. ¿Desaparecieron tres? No, lo que pasa es que las diferencias entre algunos de ellos se hicieron menos perceptibles y por eso se fusionaron. En este proceso, Arellano Marketing reasignó los individuos pertenecientes a algunos segmentos muy pequeños que no resultaban atractivos para la construcción de estrategias específicas pero respetando siempre los principales ejes que se hallaron en la clasificación de los peruanos; es decir, el eje «modernidad-conservadurismo» y «riqueza- pobreza». Así, tenemos que el 52\% de la población peruana con estilos de vida modernos se compone de la siguiente manera: $6 \%$ afortunados; $23 \%$ progresistas; y, el $23 \%$ 
modernas. Por su parte, la población con estilos de vida más conservadores, se distribuye en: $16 \%$ adaptados; $20 \%$ conservadoras; y, $12 \%$ modestos.

Sólo entre los progresistas (varones extremadamente prácticos que siempre están en busca del progreso personal y familiar) y las modernas (mujeres de empuje y trabajo que buscan la realización fuera del ámbito doméstico) se concentra el $46 \%$ de peruanos. A ellos se une el $6 \%$ de afortunados (hombres y mujeres que aspiran a diferenciarse del resto interesados en el progreso individual).

En los últimos años, se visualiza la tendencia de las empresas del área retail a reinventar su negocio en torno al cliente. El cambio tecnológico se está centrando en lo que llamamos la visión de $360^{\circ}$, donde todo gira en torno a lograr la satisfacción del cliente para conseguir que la gestión de la empresa sea exitosa. La obligación y el sueño dorado de todo retail es conocer de manera efectiva al cliente para cautivarlo.

En relación a la calidad de servicio Garza, Badii \& Abreu [4] investigaron sobre el mejoramiento de la calidad de servicios mediante el modelo de las discrepancias entre las expectativas de los clientes y las percepciones de la empresa; presentaron una caracterización general para brindar un servicio de atención al cliente de calidad, el estudio de una herramienta para analizar el mejoramiento del valor de los productos y servicios, además de una profunda reflexión sobre la conveniencia de aprovechar los conocimientos y utilizarlos en problemas de la empresa, de tal forma que permita conformar una idea más clara de la importancia y necesidad de contar con un diseño del servicio de atención al cliente; se aplico el modelo de las deficiencias en un estudio de caso de una empresa mexicana, el referido modelo está basado en parámetros de calidad y evaluación del servicio que contempla la diferencia entre las expectativas y percepciones de los consumidores fundamentado en la interrelación de factores, que ocasionan brechas en la gestión del servicio.

El objetivo general de la investigación fue determinar la relación que existe entre los estilos de vida de los clientes y la calidad de servicio de los empresarios de la ACMSB; los objetivos específicos fueron: Identificar los estilos de vida y conocer la percepción de los clientes respecto a la calidad de servicio que proporcionan los empresarios de la ACMSB. La hipótesis a demostrar es: Existe relación positiva entre los estilos de vida de los clientes y la calidad de servicio de los empresarios de la ACMSB.

\section{METODOLOGÍA}

La investigación es de enfoque cuantitativo, de tipo descriptivo correlacional, se aplico el método deductivo, busco la correlación entre estilos de vida de los clientes y calidad de servicio de los empresarios de la ACMSB.

Para determinar el tamaño de muestra, se considero como población al número promedio de clientes que visitan diariamente las instalaciones de la ACMSB, para adquirir productos o servicios de los 20 rubros que ofrecen, que ascienden a 466 (según cálculo realizado por los dirigentes).

Aplicando la fórmula, el tamaño de muestra calculada fue de 171 clientes. El método de muestreo fue el probabilístico al azar. El ámbito espacial fue la ACMSB ubicado en el distrito de San Borja de la provincia de Lima y el ámbito temporal el año 2010, los cuestionarios tanto del Servqual como el de estilos de vida debidamente fiables por haber pasado la prueba de confiabilidad Alfa de Cronbach, se aplicaron a fines del mes de julio del 2010 a los clientes que realizaban sus compras en los diversos rubros de la ACMSB.

Los participantes fueron clientes varones $y$ mujeres mayores de 18 años, que se encontraron comprando bienes y servicios de los diversos rubros de la ACMSB, cuyo consumo supero los $S / .10 .00$. Se incluyeron a las empleadas del hogar que cumplieron los requisitos antes mencionados y se excluyeron a clientes mayores de 80 años. Estos clientes se encontraron consumiendo en la ACMSB que agrupa a 200 empresarios, dedicados a la venta de diversos productos para el consumo familiar: verduras, menestras, carnes, aves, pescados, embutidos, peluquería, entre otros.

Como instrumentos se aplicaron dos cuestionarios: 1) El cuestionario sobre identificación de los estilos de vida, que consta de 6 estilos: afortunados, progresistas, modernas, adaptados, conservadores y 
modestos; agrupados en dos dimensiones: modernos y conservadores. 2) El cuestionario Servqual, de calidad de servicios constituido por 22 preguntas, de las cuales 4 están referidas a los aspectos tangibles, 5 a los aspectos de fiabilidad, 4 a sensibilidad, 4 a seguridad y 5 a empatía. La confiabilidad de la prueba se realizo mediante el Alfa de Cronbach que fue de $89 \%$.

El procedimiento para la recopilación de datos se realizo acercándose a las instalaciones de la ACMSB, en una fecha única, a fines del mes de julio del 2010, previa coordinación tanto verbal como por escrito, con los involucrados.

\section{RESULTADOS Y DISCUSIÓN}

En el cuadro $N^{0} 1$ se observa la calidad del servicio con los estilos de vida de los clientes de la ACMSB, el $23.4 \%$ de los clientes que tienen un estilo de vida moderna están totalmente satisfechos con el servicio, el $13.5 \%$ de los clientes totalmente satisfechos tienen estilos afortunado, seguido de $9.4 \%$ de clientes totalmente satisfechos con estilos progresistas, como podemos apreciar son los clientes con mayores porcentajes de satisfacción los que tienen estilo de vida moderno a diferencia de los conservadores que presentan bajo nivel de satisfacción, esto debido a que en el distrito existe un $70.2 \%$ de clientes con estilos modernos.

Cuadro $\mathbf{N}^{0} 1$

Tabla de contingencia ESTILOS DE VIDA DE LOS CLIENTES ACMSB * CALIDAD DE SERVICIO

\begin{tabular}{|c|c|c|c|c|c|c|}
\hline & & & \multicolumn{3}{|c|}{ CALIDAD DE SERVICIO } & \multirow{2}{*}{ Total } \\
\hline & & & $\begin{array}{c}\text { NO } \\
\text { SATISFECHO }\end{array}$ & $\begin{array}{l}\text { MEDIANAMENT } \\
\text { E SATISFECHO }\end{array}$ & $\begin{array}{l}\text { TOTALMENTE } \\
\text { SATISFECHO }\end{array}$ & \\
\hline \multirow{14}{*}{$\begin{array}{c}\text { ESTILOS DE } \\
\text { VIDA DE LOS } \\
\text { CLIENTES } \\
\text { ACMSB }\end{array}$} & \multirow[t]{2}{*}{ AFORTUNADAS } & Recuento & 0 & 3 & 23 & 26 \\
\hline & & $\%$ del total &, $0 \%$ & $1,8 \%$ & $13,5 \%$ & $15,2 \%$ \\
\hline & \multirow[t]{2}{*}{ PROGRESISTAS } & Recuento & 5 & 4 & 16 & 25 \\
\hline & & $\%$ del total & $2,9 \%$ & $2,3 \%$ & $9,4 \%$ & $14,6 \%$ \\
\hline & \multirow[t]{2}{*}{ MODERNAS } & Recuento & 12 & 17 & 40 & 69 \\
\hline & & $\%$ del total & $7,0 \%$ & $9,9 \%$ & $23,4 \%$ & $40,4 \%$ \\
\hline & \multirow[t]{2}{*}{ ADAPTADOS } & Recuento & 2 & 2 & 12 & 16 \\
\hline & & $\%$ del total & $1,2 \%$ & $1,2 \%$ & $7,0 \%$ & $9,4 \%$ \\
\hline & \multirow[t]{2}{*}{ CONSERVADORES } & Recuento & 0 & 4 & 13 & 17 \\
\hline & & $\%$ del total &, $0 \%$ & $2,3 \%$ & $7,6 \%$ & $9,9 \%$ \\
\hline & \multirow[t]{2}{*}{ MODESTOS } & Recuento & 3 & 5 & 10 & 18 \\
\hline & & $\%$ del total & $1,8 \%$ & $2,9 \%$ & $5,8 \%$ & $10,5 \%$ \\
\hline & \multirow[t]{2}{*}{ Total } & Recuento & 22 & 35 & 114 & 171 \\
\hline & & $\%$ del total & $12,9 \%$ & $20,5 \%$ & $66,7 \%$ & $100,0 \%$ \\
\hline
\end{tabular}

Fuente: Elaborado por la investigadora en base a los cuestionarios aplicados.

En el cuadro $\mathrm{N}^{\circ} 2$, en relación a las dimensiones de los estilos de vida con calidad de servicio, observamos que el $46.20 \%$ de los clientes totalmente satisfechos son de estilo moderno y el $20.47 \%$ de los mismos son de estilo conservador; los clientes medianamente satisfechos con estilo de vida moderna son el $14 \%$ y de estilo conservadora son el $6.4 \%$; también observamos que los clientes no satisfechos son el $12.9 \%$ de los cuales el $9.9 \%$ de estos clientes tienen un estilo de vida moderna y el $2.9 \%$ un estilo de vida conservadora. 
Cuadro $\mathbf{N}^{\circ} 2$

Tabla de contingencia CALIDAD DE SERVICIO * DIMENSIONES DE LOS ESTILOS DE VIDA

\begin{tabular}{|c|c|c|c|c|c|}
\hline & & & \multicolumn{2}{|c|}{$\begin{array}{l}\text { DIMENSIONES DE LOS } \\
\text { ESTILOS DE VIDA }\end{array}$} & \multirow[b]{2}{*}{ Total } \\
\hline & & & MODERNA & $\begin{array}{l}\text { CONSERV } \\
\text { ADORA }\end{array}$ & \\
\hline \multirow{9}{*}{$\begin{array}{l}\text { CALIDAD DE } \\
\text { SERVICIO }\end{array}$} & \multirow[t]{2}{*}{ NO SATISFECHO } & Recuento & 17 & 5 & 22 \\
\hline & & $\%$ del total & $9,9 \%$ & $2,9 \%$ & $12,9 \%$ \\
\hline & \multirow{3}{*}{$\begin{array}{l}\text { MEDIANAMENTE } \\
\text { SATISFECHO }\end{array}$} & Recuento & 24 & 11 & 35 \\
\hline & & $\%$ del total & & & \\
\hline & & & $14,0 \%$ & $6,4 \%$ & $20,5 \%$ \\
\hline & \multirow{2}{*}{$\begin{array}{l}\text { TOTALMENTE } \\
\text { SATISFECHO }\end{array}$} & Recuento & 79 & 35 & 114 \\
\hline & & $\%$ del total & $46,2 \%$ & $20,5 \%$ & $66,7 \%$ \\
\hline & \multirow[t]{2}{*}{ Total } & Recuento & 120 & 51 & 171 \\
\hline & & $\%$ del total & $70,2 \%$ & $29,8 \%$ & $100,0 \%$ \\
\hline
\end{tabular}

Fuente: Elaborado por la investigadora en base a los cuestionarios aplicados.

A continuación se analiza la relación entre la calidad de servicio y estilos de vida, para tal efecto se plantea la siguiente hipótesis estadística:

Ho: No existe relación entre los estilos de vida de los clientes y la calidad de servicio de los empresarios de la ACMSB.
Ha: Existe relación entre los estilos de vida de los clientes y la calidad de servicio de los empresarios de la ACMSB.

$H a: \rho=O$

Ho: $\rho \neq O$

Cuadro $\mathbf{N}^{\circ} 3$

Prueba de Pearson para las Correlaciones

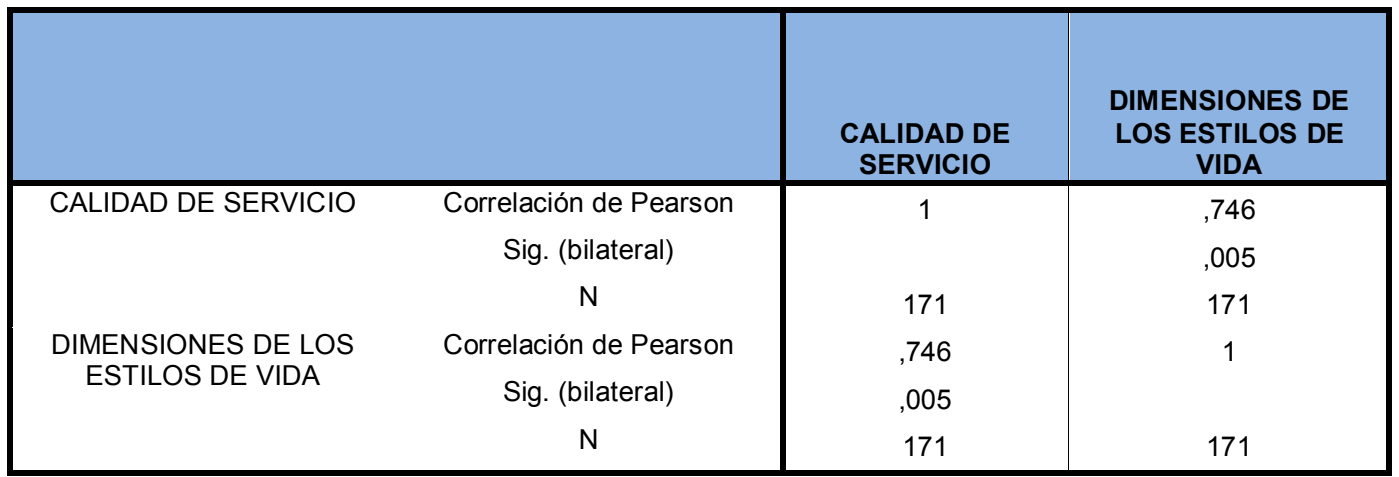

Fuente: Elaborado por la investigadora en base a los cuestionarios aplicados.

En el cuadro $\mathrm{N}^{\circ} 3$, la prueba de Pearson, arroja 0.746 , este valor significa que existe una relación de $74 \%$ entre la calidad de servicio y los estilos de vida, si observamos el Sig. (bilateral) este es de $0.005<$ 0.05 a un nivel de significancia del $5 \%$ se rechaza la hipótesis estadística, por lo que aceptamos la hipótesis alternativa. Es decir al $5 \%$ de significancia existe relación entre los estilos de vida y la calidad de servicio.

El estilo de vida de los clientes de la ACMSB es el moderno; este resultado coincide con los hallazgos 
de Arellano [1] quien reporto que el estilo de vida moderno es el preponderante en nuestro país. El hallazgo encontrado por De Soto [2] que los recién llegados del campo a la ciudad, encontraron un muro impenetrable de reglas, que los marginaban de las actividades sociales y económicas legalmente establecidas, en los que era sumamente difícil entrar a negocios formales; se ha logrado superar; puesto que, estas micro y pequeñas empresas están siendo muy bien vistos por los clientes. Mayor argumento constituye el reporte estadístico del INEI [3], que informa que la actividad comercial tuvo un crecimiento del $10,96 \%$, esto es una tendencia positiva en la mayoría de sus rubros componentes, destacándose entre otros, la venta minorista de alimentos, a través de las cadenas de supermercados e hipermercados y grandes almacenes.

\section{CONCLUSIONES}

\section{REFERENCIAS}

[1] R. Arellano, Los estilos de vida en el Perú, como somos y pensamos los peruanos del siglo XXI, Arellano investigación de Marketing S.A 2008.

[2] H. De Soto, El misterio del capital, Grupo editorial Norma S.A. (2009).

[3] Instituto nacional de estadística e informática, encuesta mensual del sector comercio (2010).

[4] Garza, Badii \& Abreu, Mejoramiento de la calidad de servicios mediante el modelo de las discrepancias entre las expectativas de los clientes y las percepciones de la empresa. En: www.daenajournal.org. Leído el 30 de marzo del 2008
1) Existe relación entre los estilos de vida de los clientes y la calidad de servicio de los empresarios de la ACMSB.

2) El estilo de vida preponderante de los clientes es el moderno.

3) La percepción de los clientes respecto a la calidad de servicio de los empresarios de la ACMSB es buena.

Se concluye que las empresas del sector retail en especial de la ACMSB, son conscientes de la invasión de las grandes cadenas de supermercados e hipermercados y grandes almacenes; por ello, están reinventando sus estrategias en torno a la satisfacción del cliente. Esta investigación servirá como base para futuras estrategias a adoptar por los empresarios de la ACMSB, a fin de mantener a los clientes satisfechos, con alta calidad de servicio.

\section{AGRADECIMIENTOS}

A los gestores de las empresas de la ACMSB y a sus clientes, por su contribución. 